\title{
Literatur
}

1. Troll, W. und R. K. Cannan, J. biol. Chemistry 200, 803 (1953). - 2. Müring, D. und E. KaIser, Hoppe-Seyler's Z. physiol. Chem. 332, 276 (1963). - 3. Constantsas, N. S. und C. Danelatou-Athanassiadou, Clin. chim. Acta (Amsterdam) 9, 1 (1964). - 4. Berger, H., M. BrenNer und H. VetterliBüchner, Aminoacidurie und Hyperaminoacidurie, Bibliotheca Paediatrica 71, S. Karger, Basel/New York (1959). - 5. Bické, H. und F. Souchon, Die Papierchromatographie in der Kinderheilkunde, (Beihefte zum Arch. Kinderhk., Stuttgart 31), Ferdi- nand Enke Verlag, Stuttgart (1955). - 6. SMrTH, J., Chromatographic Techniques, William Heinemann, London (1958). 7. Walz, D., A. R. Faymy, G. Pataki, A. NIederwieser und M. Brenner, Experientia (Basel) 19, 213 (1963). - 8. Patakr, G., Dünnschichtchromatographie in der Aminosäure- und Peptidchemie, Walter de Gruyter \& Co., Berlin (im Druck). - 9. Drrxmann, J., diese Z. 1, 190 (1963). - 10. DitrmanN, J., diese Z. 3, 59 (1965). - 11. Ditrtanan, J., J. B. Mayer und M. Wolf, Zschr. Kinderhk. 94, 130 (1965).

Dr. rer. nat. Jürgen Dittmann, Universitäts-Kinderklinik 665 Homburg-Saar

\section{Dünnschicht-Chromatographie in der Klinik}

\author{
IV. Mitteilung: Abtrennung von Phosphoaethanolamin \\ Von J. Dittmann ${ }^{1}$ ) \\ Aus der Universitäts-Kinderklinik Homburg-Saar und der Landeskinderklinik Neunkirchen/Saar-Koblbof \\ (Direktor: Prof. Dr. J.B. Mayer)
}

(Eingegangen am 22. März 1965)

\begin{abstract}
Aminosäuren können an dünnen Zelluloseschichten durch zweidimensionale Chromatographie mit folgenden Lösungsmitteln getrennt werden: Lösung a: Wasser mit $60 \mathrm{Vol} \% \mathrm{n}$-Propanol und $20 \mathrm{mMol}$ Tris-[hydroxymethyl]aminomethan, sowie $17,6 \mathrm{mMol} \mathrm{HCl} / l$; - Lösung b: Wasser mit $70 \mathrm{Vol} \%$ Isopropanol. - Entwickelt wird $10 \mathrm{~cm}$ in jeder Richtung. Phosphoaethanolamin wird unter diesen Bedingungen nicht nachweisbar zersetżt.

Amino acids can be separated on thin-layers of cellulose by 2-dimensional chromatography with the following solutions: solution a: water with $60 \mathrm{Vol} \% \mathrm{n}$-Propanol and $20 \mathrm{mMol}$ Tris-[hydroxymethyl]aminomethane and $17,6 \mathrm{mMol} \mathrm{HCl} / l$; - solution $b$ : water with $70 \mathrm{Vol} \%$ Isopropanol. - The plates are developed $10 \mathrm{~cm}$ in each direction. Under these conditions there is no detectable destruction of phosphoethanolamine,
\end{abstract}

Der Nachweis von Phosphoaethanolamin (I) in Urinproben hat Bedeutung bei der Diagnostik von Hypophosphatasie (1). Chromatographiert man saure Lösungen (2-4), die I enthalten, so erhält man 2 Flecke: I und durch Hydrolyse von I gebildetes Aethanolamin. Aus diesem Grund wurde ein gepuffertes Lösungsmittel-System geprüft, das schonende Abtrennung von I auch bei verschiedener Wasserstoffionen-Konzentration der Analysenlösung ermöglicht.

\section{Methodik}

\section{Lösung a}

$24,2 \mathrm{~g}$ Tris[hydroxymethyl]aminomethan werden in Wasser $\mathrm{zu}$ $1000 \mathrm{~m} /$ gelöst. $250 \mathrm{~m} l$ dieser Stammlösung werden mit $440 \mathrm{ml}$ $0,1 n \mathrm{HCl}$ versetzt und mit Wasser auf $1000 \mathrm{ml}$ aufgefüllt. 4 Vol. des vorstehenden Puffers werden mit 6 Vol. n-Propanol vermischt.

\section{Lösung $b$}

3 Vol. Wasser werden mit 7 Vol. Isopropanol vermischt.

Dünne Celluloseschichten werden nach beschriebener Methode (2) auf Glasplatten hergestellt. Die Analysenprobe ( $1 \mu l$ wäßrige Lösung) wird auf möglichst kleinem Fleck $17 \mathrm{~mm}$ vom unteren und $20 \mathrm{~mm}$ vom linken Rand entfernt aufgetragen. - Entwickelt wird

1) Technische Mitarbeit: G. LIEM.
$10 \mathrm{~cm}$ mit Lösung a und nach halbstündiger Zwischentrocknung bei Raumtemperatur senkrecht zur ersten Laufrichtung $10 \mathrm{~cm}$ mit Lösung $\mathrm{b}$. Nach Lufttrocknung der Rlatte wird mit Ninhydrin-Reagenz besprüht und 15 bis $30 \mathrm{Min}$. auf $70^{\circ}$ erwärmt.

\section{Ergebnisse}

$0,2 \mu \mathrm{g}$ I sind mit vorstehender Methode auf Dünnschicht-Chromatogrammen gut abtrennbar und nachweisbar. Es wird nur ein Fleck erhalten, dessen $R_{k^{-}}$ Werte sich von denen des Aethanolamins stark unterscheiden. Auch die Farbe, die man aus I und Ninhydrinreagenz nach Erwärmen erhält, unterscheidet sich von der Farbe der meisten Amine und Aminosäuren durch das Fehlen einer Rot-Komponente im Blau. Eine Verwechslungsmöglichkeit hinsichtlich $R_{F}$-Wert und Farbe des I-Flecks besteht nur bei Vorkommen von Asparaginsäure. Zur Prüfung der Leistungsfähigkeit der beschriebenen Methode wurden 5 Mischungen („A, B, C, $\left.D, E^{\prime \prime}\right)$ trennbarer Aminosäuren, Amide und Amine in Wasser hergestellt und je $4 \mathrm{mal}$ chromatographiert. Die unteren Grenzen der Nachweisbarkeit und die $R_{F^{-}}$ Werte sind in nachstehender Tabelle zusammengestellt. Hinter den $R_{F}$-Werten ist jeweils die mittlere Streuung der Einzelwerte um den Mittelwert angegeben. 
Tab. 1

$R_{F}$-Werte an Cellulose-Schichten

$R_{F_{1}}$ : Chromatographie mit Isg. a; $R_{F_{2}}$ : Chromatograghie mit Lsg. b; (siche „Methodik“)

\begin{tabular}{|c|c|c|c|c|}
\hline Substanz & $\begin{array}{l}\text { Menge } \\
(\mu \mathrm{g})\end{array}$ & $R_{F_{1}}$ & $R_{F_{2}}$ & $\begin{array}{l}\text { chromato- } \\
\text { graphiert } \\
\text { in } \\
\text { Gemisch }\end{array}$ \\
\hline L-Ornithin & 0,08 & $0,33 \pm 0,05$ & $0,09 \pm 0,01$ & $\mathbf{A}$ \\
\hline L-Glutamin & 0,4 & $0,35 \pm 0,02$ & $0,10 \pm 0,02$ & B \\
\hline L-Asparagin & 0,5 & $0,35 \pm 0,02$ & $0,23 \pm 0,02$ & B \\
\hline $\begin{array}{l}\text { L-Histidin } \\
\text { DL-1-Methylhistidin }\end{array}$ & $\begin{array}{l}0,1 \\
0,1\end{array}$ & $0,36 \pm 0,01$ & $0,10 \pm 0,01$ & $\mathrm{E}$ \\
\hline $\mathrm{L}$-Arginin & 0,3 & $0,38 \pm 0,01$ & $0,13 \pm 0,01$ & D \\
\hline L-Asparaginsäure & 0,1 & $0,39 \pm 0,02$ & $0,27 \pm 0,01$ & C \\
\hline Histamin & 0,15 & $0,41 \pm 0,03$ & $0,14 \pm 0,04$ & C \\
\hline DL-Lysin & 0,08 & $0,41 \pm 0,02$ & $0,30 \pm 0,02$ & B \\
\hline Phosphoaetbanolamin & 0,2 & $0,42 \pm 0,03$ & $0,28 \pm 0,04$ & D \\
\hline L-Citrullin & 0,2 & $0,42 \pm 0,03$ & $0,28 \pm 0,01$ & A \\
\hline DL-Serin & 0,1 & $0,44 \pm 0,02$ & $0,34 \pm 0,02$ & B \\
\hline Glycin & 0,05 & $0,44 \pm 0,03$ & $0,34 \pm 0,05$ & $\mathrm{D}$ \\
\hline L-Glutaminsäure & 0,1 & $0,48 \pm 0,03$ & $0,41 \pm 0,05$ & $\mathrm{D}$ \\
\hline L-Hydroxyprolin & 0,5 & $0,49 \pm 0,03$ & $0,40 \pm 0,03$ & A \\
\hline Taurin & 0,1 & $0,50 \pm 0,02$ & $0,45 \pm 0,03$ & E \\
\hline D-Glucosamin & 0,4 & $0,51 \pm 0,02$ & $0,51 \pm 0,02$ & B \\
\hline Sarkosin & 0,5 & $0,51 \pm 0,03$ & $0,43 \pm 0,02$ & B \\
\hline L-Alanin & 0,08 & $0,52 \pm 0,03$ & $0,46 \pm 0,03$ & A \\
\hline$\beta$-Alanin & 0,1 & $0,53 \pm 0,06$ & $0,51 \pm 0,04$ & A \\
\hline L-Threonin & 0,2 & $0,54 \pm 0,03$ & $0,45 \pm 0,01$ & C \\
\hline L-Prolin & 0,8 & $0,57 \pm 0,04$ & $0,50 \pm 0,04$ & A \\
\hline$\delta$-Aminolaevulinsäure & e 0,5 & $0,62 \pm 0,03$ & $0,61 \pm 0,07$ & $\mathrm{D}$ \\
\hline Aetbanolamin & 0,05 & $0,63 \pm 0,05$ & $0,63 \pm 0,06$ & D \\
\hline DL-Tyrosin & 0,2 & $0,63 \pm 0,03$ & $0,56 \pm 0,03$ & $\mathbf{E}$ \\
\hline $\begin{array}{l}\text { DL- } \beta \text {-Amino-n- } \\
\text { buttersäure }\end{array}$ & 0,5 & $0,64+0,02$ & $\pm 0,02$ & C \\
\hline $\begin{array}{l}\beta \text {-Amino-iso-butter- } \\
\text { säure }\end{array}$ & 0,1 & $0,07 \perp 0,0$ & $\perp 0,00$ & 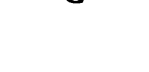 \\
\hline $\begin{array}{l}\text { DL- } \alpha \text {-Amino-n- } \\
\text { buttersäure } \\
\alpha \text {-Amino-iso-butter- }\end{array}$ & 0,1 & & & \\
\hline $\begin{array}{l}\alpha \text {-Amino-1so-butter- } \\
\text { säure } \\
\gamma \text {-Amino-n-butter- } \\
\text { säure }\end{array}$ & 0,2 & $0,64 \pm 0,03$ & $0,69 \pm 0,05$ & $\mathrm{E}$ \\
\hline DL-Tryptophan & 0,2 & $0,64 \pm 0,03$ & $0,54 \pm 0,06$ & $\mathrm{D}$ \\
\hline DL-Valin & 0,05 & $0,66 \pm 0,03$ & $0,64 \pm 0,03$ & A \\
\hline DL-Methionin & 0,1 & $0,68 \pm 0,02$ & $0,65 \pm 0,02$ & B \\
\hline DL-Norvalin & 0,15 & $0,69 \pm 0,02$ & $0,69 \pm 0,05$ & A \\
\hline DL-Phenylalanin & 0,4 & $0,74 \pm 0,02$ & $0,69 \pm 0,03$ & B \\
\hline 3.5-Dijod-L-tyrosin & 0,8 & $0,76 \pm 0,03$ & $0,69 \pm 0,06$ & $\mathrm{E}$ \\
\hline DL-Isoleucin & 0,05 & $0,79 \pm 0,02$ & $0,81 \pm 0,02$ & C \\
\hline DL-Leucin & 0,05 & $0,84 \pm 0,03$ & $0,84 \pm 0,01$ & C \\
\hline
\end{tabular}

\section{Diskussion}

Gegenüber der Trennung von sauren AminosäureLösungen mit Propanol-Wasser und IsopropanolWasser $(2-4)$ hat die hier beschriebene Methode drei Nachteile:

1. Die Dünnschicht färbt sich bei längerem Erhitzen und auch bereits bei mehr als eintägiger Lagerung infolge der Beladung mit "Tris" und dem aus „Tris" mit Ninhydrinreagenz entstehenden gefärbten Stoff in bestimmten Bereichen gelb und in anderen Bereichen bläulich an; Auswertung der Chromatogramme sollte deshalb wenige Stunden nach Anfärbung erfolgen.

2. Die Streuung der $R_{Y^{-}}$Werte, insbesondere auch die des $R_{F}$-Wertes von $\mathrm{I}$, ist hoch. Eine wesentliche Ursache scheint unterschiedliche Dicke der Zellulose-Schichten zu sein. Man kann die Streuung kleiner halten, wenn man die Schichten unter möglichst konstanten Bedingungen (Streichgeschwindigkeit bei Verwendung eines Streichgerätes) herstellt. Die in der Tabelle angegebenen $R_{F}$-Werte wurden an Platten ermittelt, die nicht gemeinsam beschichtet worden waren.

3. Die unteren Grenzen der Nachweisbarkeit liegen meistens etwas höher als im ungepufferten System $(2-4)$.

Der Vorteil der Methode liegt dagegen darin, daß die Hydrolyse von I wegen des fast neutralen $\mathrm{pH}$-Wertes sehr klein gehalten wird und man aus I nur einen Fleck erhält. - Soll I in biologischen Flüssigkeiten nachgewiesen werden, so kann man, wie bereits beschrieben (4), die Analysenflüssigkeit ohne Aufarbeitung direkt auf die Platte auftragen und dann chromatographieren. Zur Erhöhung der Zuverlässigkeit der Methode empfiehlt es sich, auf einer zweiten Platte die gleiche Menge Analysenflüssigkeit plus $0,1 \mu \mathrm{g} I$ aufzutragen und zu sehen, ob man auf dieser Platte nach Entwickeln und Anfärben an der Stelle, wo man nach den $R_{F^{-}}$ Werten I vermuten kann, einen oder zwei Flecke erhält.

Ein weiterer Vorteil des beschriebenen Systems beruht in der Möglichkeit, die gleiche Analysenprobe in verschiedener Weise auf Dünnschichtplatten zu entwickeln und dadurch auch die Sicherheit des Nachweises anderer Aminosäuren zu erhöhen.

\section{Literatur}

1. Smirth, I., Chromatographic Techniques, S. 108, William Heinemann, London (1958). - 2. DitrmanN, J., diese Z. 1, 190 (1963),
- 3. Dirtmann, J., diese Z. 3, 59 (1965). - 4. Ditrmann, J., diese Z. 4, 8 (1965).
Dr. rer. nat. Jürgen Dittmann Universitäts-Kinderklinik 665 Homburg-Saar 